\title{
LA SECTION DE LANGUE FRANÇAISE DE LONDRES
}

M. Molnar vient de nous dire quelles difficultés il y avait à étudier la proscription française à Londres, en Angleterre, combien peu de progrès - à notre connaissance - avaient été faits en ce sens, jusqu'à ce jour. Quel guêpier ici en effet! les témoignages de Marx, de Vermersch, qui pourtant ne s'aimaient guère, s'accordent sur ce point. ${ }^{1} \mathrm{Il}$ arrive quelquefois que la fortune se montre secourable à l'historien. Nous avons retrouvé, égaré dans les Papiers Descaves de l'I.I.S.G., ${ }^{2}$ un document qui ne nous semble pas de médiocre importance: la délégation donnée à $\mathbf{B}$. Le Moussu par la «Section de Langue Française de Londres", pour la représenter au Congrès de La Haye. Nous le livrons au lecteur, et surtout au chercheur, sous sa forme brute avec le minimum de commentaires.

Section «de Langue Française»! Il ne s'agit pas de la "Section française» ou "Section fédéraliste de 1871", connue plus tard sous le nom de la "Parisienne», formée en septembre 1871 par des Internationaux proscrits, et qui eut de graves démêlés avec le Conseil général de Londres, celui-ci refusant, pour des raisons sur lesquelles il y aurait lieu de se pencher attentivement, son affiliation à l'A.I.T. ${ }^{3}$ La Section de Langue Française est une section, adversaire de la précédente, que nous connaissions à peine, par de vagues allusions faites dans les Minutes du Conseil général. 16 janvier 1872: "The Chairman announced that a

1 Vermersch, lettre à Vuillaume du 12 août 1872 , citée dans M. Vuillaume, Mes Cahiers rouges, X. - Proscrits, p. 35: "Ici c'est un pêle-mêle, un tohu-bohu, une confusion, une cour du roi Pétaud à faire se tordre de rire un caricaturiste [...] Naturellement celui-ci exclut celui-là qui fulmine contre cet autre qui appelle ce quatrième mouchard qui traite le cinquième de traitre qui demande des comptes à ce sixième qui ayant emporté la caisse boit, mange, rigole et se fout des cinq autres comme de l'an quarante..." Rangera-t-on déjà parmi les membres de cette "cour du roi Pétaud" les adhérents de notre Section de Langue Française?

2 Dossier Bourdeille.

3 Sur cette section et la querelle qui l'opposa au Conseil général, voir tout particulièrement A. Lehning, Archives Bakounine, t. II, Michel Bakounine et les conflits dans l'Internationale, 1872, pp. 442-451, notes 297 à 300 . 
communication had been received from the new French-speaking branch, notifying that Citizens De Wolfers and Margueritte had been appointed as the delegates of the section and asking the General Council to accept them. It was proposed [...] that they become members of the Council. The proposition was carried unanimously. "1 6 fevrier: "The rules of a newly formed French section were sent into the Council for ratification, and were referred to the Rules Committee.» ${ }^{2}$ Ensuite les Minutes ne nous donnent plus guère de détails (le règlement de la section semble toutefois avoir été accepté); on peut constater seulement la présence assez régulière au Conseil général de Marguerittes, jusqu'au Congrès de La Haye. Dans la brochure Les prétendues scissions dans l'Internationale, les auteurs, après avoir conté leurs querelles avec la première section française, ajoutent: «De l'autre côté, la masse des réfugiés français à Londres a formé une section qui est en harmonie complète avec le Conseil général.»3 Selon Engels, dans une lettre à Lafargue du 19 janvier 1872, la section "orthodoxe" aurait compté soixante membres. ${ }^{4}$

Le document que nous présentons apporte ici quelques précisions. C'est le 19 novembre qu'aurait été fondée la section. Au «mieux de sa forme", si l'on peut dire, elle aurait eu 79 membres. Mais le 30 ou le 31 août 1872, ils ne sont en réalité que 34 à donner délégation à Le Moussu; 27 ont quitté Londres, soit pour l'étranger, soit pour Middlesbrough dans le Yorkshire (Milesboro dans le texte), l'une des sections du British Federal Council; manquent 18 inscrits dont les noms ne sont pas indiqués, ni leur destin - sans doute se sont-ils retirés ou ontils été exclus. Le secrétaire est Edouard Bourdeille (membre également de la Société des Réfugiés, comme, semble-t-il, la plupart des adhérents), commis-voyageur, né à Paris le 9 février 1842, qui appartenait à l'Internationale dès avant la Commune: dans les parcours en France que lui imposait son métier, il répandait le journal La Marseillaise, indiquait les contacts révolutionnaires qu'il avait pu établir. ${ }^{5}$

Il est à souligner qu'aucun des 79 n'a appartenu préalablement à la première "Section française». Nous sommes bien dans deux camps différents, et tandis que la première section comptait ce qu'on pourrait

1 Minutes 1871-1872, pp. 77-78. Voir aussi la séance du 21 novembre 1871, p. 41.

2 Ibid., p. 96. C'est à tort, me paraît-il, que les éditeurs russes des Minutes considèrent les statuts dont il est fait ici mention, comme ceux d'une nouvelle, d'une troisième section française. De Wolffers, qui n'est pas porté sur la liste de la section a démissionné du Conseil général le 27 juillet 1872 : ibid., p. 314.

s J. Freymond, Recueil, t. II, p. 280.

4 Correspondance F. Engels - P. et L. Lafargue, Editions Sociales, Paris 1956,

t. I, p. 20.

- A.H.G., pièce non cotée: Correspondance du journal La Marseillaise. 
appeler le "gratin" des sections parisiennes, ${ }^{1}$ on ne trouve ici que du menu fretin de la Commune, à quelques exceptions près: Emile Bauche, membre du Comité de vigilance et de la Commission militaire du VIIIe arrondissement, Léon Landrin, du syndicat des ouvriers du Bronze, Désiré Lapie, du Comité de vigilance du XVIIIe (comme aussi Bourdeille), Ladislas de Kuksz, "Directeur des transmissions et de la Télégraphie», Marguerittes, chef du 69e bataillon..., ${ }^{2}$ et naturellement Serraillier et son ami Rochat (ce dernier parti pour la Belgique le 30 mai 1872), Frankel, Le Moussu, également du Comité du XVIIIe et commissaire de police en 1871. L'affiliation de ces quatre hommes, ainsi que celle de Hermann Jung, montre bien qu'on est en présence de la section marxienne "orthodoxe" par excellence. Au Congrès de La Haye, dont il sera le secrétaire pour le français, Le Moussu, l'élu, votera toujours avec la plus grande fidélité dans la ligne du "parti Marx.»"

1 Voir la liste des noms des adhérents de celle-ci dans A. Lehning, op. cit.: Avoine fils, Avrial, A. Barré, Bastelica (Marseille), V. Bonneaure, Bossens, Cirode (section de Vaugirard), Chalain (Vaugirard puis Batignolles), J.-B. Chautard (Sociale des Ecoles), Davoust (Batignolles), Doby (Batignolles), A. Dupont (Batignolles?), G. Durand (Chambre syndicale des Bijoutiers), Gobert (XIIIe arrondissement), Husson, B. Landeck (Sociale des Ecoles), Laroque, E. Leverdays, Lohay, A. Lonclas, Magdonel (Faubourg-du-Temple), Maréchal, L. A. Moreau (Batignolles), Neveux (XIIIe), Ch. Niémers (Sociale des Ecoles), J. Olivier (Batignolles), H. Piednoir (Gravilliers), E. Roullier (Sociale des Ecoles), Schmeltz (Malesherbes), L. Sornet, Tanguy (Gravilliers), Theisz (Bronziers et Chambre fédérale), E. Vermersch, Wauthier, I. Wolff.

2 On doit pouvoir aussi compter G. Bertin (no 44), qui est selon toute probabilité l'ancien secrétaire de la Commission du Travail et de l'Echange (mais il est dit ici peintre, alors que - s'il s'agit du même - sa profession en 1871 était celle de lithographe), Alexis Dardelle, colonel d'Etat-major. On trouvera dans J. Maitron, D.B.M.O.F., les biographies de: E. Bauche, Beucher, Th. Blond, Brignolas, E. Bourdeille, Bugnon, G. Carnovale, G. Chaboud, Chol (militant lyonnais bien connu), A. Dardelle, L.-J. Delhaye, Fondeville, Gourlet, L. de Kuksz, D. Lapie, Le Moussu, Lebas fils, Marguerittes, N. Maujean, D. Parigot, J. Rinck, Valeille, sans compter naturellement Frankel, Rochat et Serraillier. Avaient assisté, au titre de "visiteurs", aux séances du Conseil général de juin et juillet 1871 un de Baufort, qui pourrait bien être le no 32 , Beaufort, Légé, peut-être le même que le no 55 Léger, et Rovart, no 33. Selon J. Maitron, D.B.M.O.F., Rovart serait le pseudonyme ou le surnom de J. Pauvert, Directeur de la Télégraphie pendant la Commune; mais ce dernier était employé au télégraphe de son métier, alors qu'ici Rovart est désigné comme étudiant en droit.

3 Voir J. Freymond, Recueil, t. II, pp. 329-330, la liste de délégués au Congrès, et, passim, les interventions de ceux qui nous intéressent: en effet parmi les membres de la Section de Langue Française sont, outre Le Moussu, délégués au Congrès Frankel, "pour une section française", et Serraillier, "pour le Conseil général". On a pu prétendre que Marx avait "fabriqué" sa majorité au Congrès. Ne demandait-il pas à Sorge, le 21 juin 1872, des mandats, en blanc en somme, pour les Allemands, les Irlandais, «Les Français [...] G. Ranvier, Auguste Serraillier, Le Moussu, Ed. Vaillant, F. Cournet, Ant. Arnaud "? (Correspondance 
Qu'advint-il par la suite de la section? Il paraît probable qu'elle s'est disloquée après La Haye. Dès les lendemains de La Haye, et bien que, semble-t-il, il n'y ait pas assisté, Marguerittes, représentant de la section auprès du Conseil général, signait la brochure blanquiste Internationale et Révolution; il sera également, avec Aberlen ( $\left.\mathrm{n}^{\circ} 77\right)$, l'un des signataires de la proclamation Aux Communeux, lancée en 1874 par le groupe "La Commune révolutionnaire» blanquiste. Dardelle, Maujean, Rinck signent de leur côté la brochure $A$ la Classe ouvrière, publiée le 1 er octobre 1874 par le «Comité révolutionnaire du prolétariat" de V. Delahaye (à ne pas confondre avec le Delhaye, no 68 de la liste). On sait que $H$. Jung a rompu avec Marx dès décembre 1872 pour rejoindre la fraction sécessionniste du British Federal Council. Serraillier et Le Moussu assisteront encore au Congrès de Manchester ( $1^{\mathrm{er}}-3$ juin 1873) de la fraction fidèle à Marx du Conseil Fédéral Anglais. Mais ensuite Marx n'a pas tardé à rompre avec Le Moussu, comme en témoigne sa lettre à Sorge, le 4 août 1874: «Les quelques Français (j'entends de ceux qui tenaient encore avec nous à la Haye) se sont pour la plupart révélés ensuite fripouilles, notamment M. Le Moussu, qui m'a filouté, ainsi que d'autres, pas mal d'argent et a ensuite cherché par d'infâmes calomnies à se blanchir en belle âme méconnue.. ${ }^{\mathbf{1}}$ La rupture avec Serraillier, naguère tant apprécié, qui est encore le délégué de Londres au VIe Congrès de l'A.I.T. "orthodoxe" à Genève (septembre 1873), n'a pas dû être beaucoup plus tardive. Son oraison funèbre, c'est Jenny Marx qui la prononce dans sa lettre à Sorge du 21 janvier 1877: "[...] il y en a quelques-uns que nous ne voyons plus, notamment plus

Fr. Engels - K. Marx et divers. Publiée par F. A. Sorge, Costes, Paris 1950, t. I, p. 87). Il faut souligner que dans le cas de Le Moussu au moins, il y a délégation régulière d'une section. Au Congrès, Le Moussu s'est fait violent accusateur des "Jurassiens»; il s'est prononcé pour l'exclusion de Bakounine, de Guillaume et de Schwitzguébel; il a signé la motion contre l'abstention en matière politique, voté les articles 2 et $7 \mathrm{a}$, voté également le déplacement du Conseil général à New York; bref, et en tous points, l'orthodoxie incarnée. La suite va montrer qu'elle fut mal récompensée.

1 Correspondance Fr. Engels - K. Marx et divers, op. cit., t. I, p. 200. Dans sa lettre à Sorge des 12-17 septembre 1874, ibid., p. 207, Engels est plus explicite encore et étend le réquisitoire à toute l'émigration française: "L'émigration française est tout à fait divisée; ils se sont tous brouillés entre eux, et avec tout le monde du reste, pour des motifs purement personnels, affaires d'argent presque toujours, et nous sommes à peu près débarrassés d'eux. Ces gens voudraient tous vivre sans travailler réellement, ont la tête pleine de prétendues inventions qui doivent produire des millions [...]. Mais si quelqu'un est assez simple pour marcher, il est refait de son argent et par-dessus le marché traité de bourgeois. Celui qui s'est comporté le moins proprement est Le Moussu, qui s'est révélé escroc. La vie de bohême menée durant la guerre, la Commune et l'exil, a cruellement démoralisé ces gens..." Belle ressemblance avec l'appréciation de Vermersch, citée plus haut! 
de Français: pas de Le Moussu, pas de Serraillier, surtout pas de blanquistes. We had enough of them....11 Mais il $\mathrm{y}$ a probablement grand temps déjà que la Section de Langue Française de 1871 s'est volatilisée.

\title{
DOCUMENT
}

\author{
Délégation Le Moussu (Benjamin-Constant) \\ Dessinateur Industriel \\ 26, Howland S[tree]t, Fitzroy Sq[ua]re \\ Londres
}

\section{Section de Langue Française de Londres}

La Section de Langue Française [de] Londres, réunie en Assemblée extraordinaire le 28 août 1872, - a délégué le citoyen Le Moussu (Benjamin Constant) au Congrès de l'Association Internationale des Travailleurs qui se tiendra à La Haye, le deux septembre $1872 \&$ jours suivants.

Le citoyen Le Moussu a pour mission spéciale de présenter au Congrès, les révisions \& modifications jugées nécessaires par la Section aux Statuts généraux de l'Association. Le mandat qui lui est donné à ce sujet est absolument impératif.

En conséquence de cette délégation le citoyen Le Moussu (B.C.) présentera ce pouvoir à qui de droit \& prendra part aux travaux du Congrès.

Salut \& Egalité.

Londres, 30 août 1872 .

Pour la Section \& par ordre: Le secrétaire

Edouard Bourdeille

1 Ibid., t. I, p. 227. Il y a là probablement encore une affaire d'argent, qui semble née des difficultés qu'on rencontra à rembourser à Serraillier ses frais lorsqu'on l'envoya au Congrès de Genève de 1873. L'argent toujours! Et, semble-t-il, dès 1871 : on ne peut s'empêcher de penser à ce passage de la réponse d'E. Teulière, membre de la section française de 1871 (la première) en réponse aux "Prètendues scissions": "Après le grand désastre, nous arrivons à Londres meurtris, mais pleins d'espoir. Nous espérions y trouver des amis comme nous en avions trouvé en Belgique. Notre espoir fut de courte durée. Notre réception à Holborn fut mauvaise. Le mot de mendiant fut même prononcé par K. Marx et vivement relevé par l'un de nous, Roullier...", reproduit in J. Freymond, Recueil, t. II, p. 297. - En ce qui concerne cependant Serraillier plus précisément, peut-être ses difficultés avec Marx ont-elles commencé dès La Haye, où, si l'on en croit J. Guillaume, quelques heurts auraient déjà eu lieu en coulisse entre les deux hommes (J. Guillaume, L'Internationale...., t. II, p. 327). Selon ce dernier, Serraillier aurait forcé Marx, par des moyens qui n'étaient peut-être pas très loyaux, à accepter un départ de Londres du Conseil général. Cela ne concorde guère avec ce que nous savons de l'attitude de Marx sur ce point. Mais n'est-il pas possible que Guillaume ait interprété de travers une querelle qui déjà s'amorçait? 
Noms des membres composant la section de Langue fse fondée à Londres, le 19. 9 bre. 1871 -

1. Bauche (Emile), comptable.

2. Bugnon - peintre décorateur.

3. Chambault - ciseleur.

6. Gourlet (Léon), commis libraire.

11. Frankel (Léo), bijoutier.

14. Brignollas (Michel), tapissier [lire Brignolas].

19. Landrin, homme de peine.

20. Rouch, petit marchand.

24. Thévenon, Barthélemy, mécanicien.

30. Serraillier (Auguste), profess ${ }^{r}$. de français, ex-ouvrier formier.

31. Jung (Hermann) - horloger.

33. Rovart (Gabriel) - étudiant en droit.

34. Lebas fils, employé.

35. Bourdeille (Edouard) - commis libraire.

36. Chol - cordonnier.

37. citoyenne Chol - ouvrière mécanicienne.

38. Fondeville (négociant).

39. Le Moussu, dessinateur industriel.

40. Marguerittes - sans profession.

41. Richard (Victor) - négociant.

44. Bertin (Georges), peintre.

54. Dardelle (Alexis) - professeur de musique.

58. Lapie (Désiré), menuisier.

61. citoyenne Thévenon, cuisinière.

68. Delhaye (L. J.), ex-employé.

69. Blond (Théodule), doreur sur métaux.

70. Chaboud (Gustave), ciseleur.

71. Rinck (Joseph), fleuriste artificiel.

73. Parigot (Désiré), fleuriste artificiel.

75. Maujean (Nicolas), menuisier en pianos.

76. Beucher - mécanicien.

77. Aberlen (F. J.), zingueur.

78. Péssard - homme de peine.

79. Campbell (Duncan), comptable (anglais).

Citoyens faisant toujours partie de la Section, mais ayant quitté Londres

4. Durut (comptable), maintenant en Belgique.

8. Potigny (Paul), chaudronnier - maintenant en Ecosse.

15. Forest (Armand), employé en Sicile à Palerme.

16. Déthiange, tapissier.

17. Kuskz (Ladislas), prof[esseu]r de français en Ecosse.

18. Gagneux - cordonnier.

9. Valeille.

25. Caillaux Pierre - homme de peine - à Milesboro, Angleterre.

26. Jaudin (Etienne) en Amérique, boucher.

27. Griveaux.

28. Favenis.

32. Beaufort - à Preston (Angleterre).

43. Rochat (Ch.), Belgique.

47. Nicolas - Belgique. 
52. François (J.) do.

53. cit[oyen]ne Cailleaux à Milesborough (Angleterre).

55. Léger (tailleur), Angleterre.

56. Lequen (Claude), tailleur de pierres à Milesborough.

57. cit $^{\text {ne }}$ Griveaux.

59. Carnovale à Milesboro.

60. citoyenne Carnovale à Milesboro.

62. citoyenne Durut en Belgique.

63. Baudaux à Milesboro.

64. Pennissardo à Milesboro.

65. Salnier $d^{\circ} d^{0}$.

66. Journier do do.

67. Clairet (Jules) do do.

Certifié conforme au Tableau des membres de la Section de Langue française de Londres.

Londres, 31 août 1872.

Pour la section:

Le secrétaire

[signature] Edouard Bourdeille 